\title{
Respiratory Impairment Predicts Response to IL-1 and IL-6 Blockade in COVID-19 Patients With Severe Pneumonia and Hyper-Inflammation
}

\section{OPEN ACCESS}

Edited by:

Xulin Chen,

Jinan University, China

Reviewed by:

Domenico Mavilio,

University of Milan, Italy

Amy L. MacNeill,

Colorado State University,

United States

*Correspondence:

Emanuel Della-Torre

dellatorre.emanuel@hsr.it

Lorenzo Dagna

dagna.lorenzo@hsr.it

${ }^{\dagger}$ These authors have contributed equally to this work

Specialty section: This article was submitted to

Viral Immunology,

a section of the journal

Frontiers in Immunology

Received: 03 March 2021 Accepted: 16 April 2021

Published: 29 April 2021

Citation:

Della-Torre E, Lanzillotta $M$, Campochiaro C, Cavalli G, De Luca G, Tomelleri A, Boffini N, De Lorenzo R,

Ruggeri A, Rovere-Querini $P$, Castagna A, Landoni $G$, Tresoldi $M$, Ciceri F, Zangrillo A and Dagna L

(2021) Respiratory Impairment Predicts Response to IL-1 and IL-6

Blockade in COVID-19 Patients

With Severe Pneumonia and Hyper-Inflammation.

Front. Immunol. 12:675678. doi: 10.3389/fimmu.2021.675678
Emanuel Della-Torre ${ }^{1,2^{*}+}$, Marco Lanzillotta ${ }^{1+}$, Corrado Campochiaro ${ }^{1,2}$, Giulio Cavalli ${ }^{1,2}$, Giacomo De Luca ${ }^{1,2}$, Alessandro Tomelleri ${ }^{1,2}$, Nicola Boffini ${ }^{1,2}$, Rebecca De Lorenzo ${ }^{1}$, Annalisa Ruggeri ${ }^{1,3}$, Patrizia Rovere-Querini ${ }^{1,4}$, Antonella Castagna ${ }^{1,5}$, Giovanni Landoni ${ }^{1,6}$, Moreno Tresoldi ${ }^{7}$, Fabio Ciceri ${ }^{1,3}$, Alberto Zangrillo ${ }^{1,6}$ and Lorenzo Dagna ${ }^{1,2^{*}}$ for the BIO-RAF Study Group

1 Università Vita-Salute San Raffaele, IRCCS San Raffaele Scientific Institute, Milan, Italy, ${ }^{2}$ Unit of Immunology, Rheumatology, Allergy and Rare Diseases, IRCCS San Raffaele Scientific Institute, Milan, Italy, ${ }^{3}$ Hematology and Bone Marrow Transplant Unit, IRCCS San Raffaele Scientific Institute, Milan, Italy, ${ }^{4}$ Division of Immunology, Transplantation and Infectious Diseases, IRCCS San Raffaele Scientific Institute, Milan, Italy, ${ }^{5}$ Department of Infectious Diseases, IRCCS San Raffaele Scientific Institute, Milan, Italy, ${ }^{6}$ Department of Anesthesia and Intensive Care, IRCCS San Raffaele Scientific Institute, Milan, Italy, ${ }^{7}$ General Medicine and Advanced Care Unit, IRCCS San Raffaele Scientific Institute, Milan, Italy

Background: Restraining maladaptive inflammation is considered a rationale strategy to treat severe coronavirus disease-19 (COVID-19) but available studies with selective inhibitors of pro-inflammatory cytokines have not provided unequivocal evidence of survival advantage. Late administration is commonly regarded as a major cause of treatment failure but the optimal timing for anti-cytokine therapy initiation in COVID-19 patients has never been clearly established.

Objectives: To identify a window of therapeutic opportunity for maximizing the efficacy of interleukin (IL)-1 and IL-6 blockade in COVID-19.

Methods: Survival at the longest available follow-up was assessed in severe hyperinflamed COVID-19 patients treated with anakinra, tocilizumab, sarilumab, or standard of care, stratified according to respiratory impairment at the time of treatment initiation.

Results: 107 patients treated with biologics and 103 contemporary patients treated with standard of care were studied. After a median of 106 days of follow-up (range 3-186), treatment with biologics was associated with a significantly higher survival rate compared to standard therapy when initiated in patients with $\mathrm{PaO}_{2} / \mathrm{FiO}_{2} \geq 100 \mathrm{mmHg}(\mathrm{p}<0.001)$. Anakinra reduced mortality also in patients with $\mathrm{PaO}_{2} / \mathrm{FiO}_{2}<100 \mathrm{mmHg}(\mathrm{p}=0.04)$.

Conclusions: IL-1 and IL-6 blocking therapies are more likely to provide survival advantage in hyper-inflamed COVID-19 patients when initiated before the establishment of severe respiratory failure.

Keywords: COVID-19, SARS-CoV-2, interleukin-6, interleukin-1, sarilumab, anakinra, tocilizumab 


\section{INTRODUCTION}

Life-threatening coronavirus disease-19 (COVID-19) is sustained by a maladaptive inflammatory response induced by the Severe Acute Respiratory Syndrome Coronavirus 2 (Sars-CoV-2) $(1,2)$. This hyper-inflammatory response resembles the "cytokine storm" observed during macrophage-activation syndrome and is characterized by excessive release of pro-inflammatory cytokines including interleukin (IL)-1, IL-6, tumor necrosis factor, and granulocyte-macrophage colony stimulating factor, among others $(1,2)$.

Based on this hyper-inflammatory pathogenic background, targeting upstream molecules in the inflammatory cascade such as IL-1 and IL-6 soon appeared as a promising therapeutic strategy to contrast the progression of severe COVID-19, and high hopes were placed on the IL-1 and IL- 6 blocking agents anakinra, tocilizumab, and sarilumab (3-10). In particular, anakinra is a recombinant replica of the IL-1 receptor antagonist that binds to soluble IL- $1 \alpha$ and IL- $1 \beta$ preventing their pro-inflammatory activity (10). Tocilizumab and sarilumab, on the other hand, are humanized monoclonal antibodies that block IL-6-mediated signal transduction by targeting both the membrane and soluble forms of the IL-6 receptor (3). Yet, despite great expectations and encouraging outcomes from preliminary observational cohorts, tocilizumab and sarilumab lately failed to demonstrate an impact on disease mortality in open-label and randomized clinical trials (38). Similarly, recently published controlled investigations with anakinra also returned an overall unclear survival benefit compared to supportive therapies (10-12). Of note, non-response to IL- 1 and IL- 6 blocking therapies has been commonly attributed to late administration of these agents at advanced disease stage but the optimal timing of anti-inflammatory treatment initiation in COVID-19 has never been clearly established.

During the first COVID-19 surge that stroke Lombardy region (Italy) in the spring of 2020, our Institute hospitalized 954 patients and rapidly set in place contemporary open label observational studies of anakinra, sarilumab and tocilizumab to treat severe hyper-inflamed COVID-19 patients fulfilling common sets of inclusion criteria $(3,4,9,10,13,14)$. This unique experience on a homogeneous population cohort now provides an unprecedented occasion to address clinical and serological variables associated with COVID-19 response to anti-cytokine therapies, and to identify a "window of therapeutic opportunity" for maximizing the efficacy of IL-1 and IL- 6 blockade in these patients.

\section{METHODS}

\section{Study Population and Eligibility Criteria}

This study was conducted from February $25^{\text {th }} 2020$, through May $20^{\text {th }} 2020$ at San Raffaele Hospital (Milan, Italy) during the first COVID-19 outbreak in Lombardy region. Hospitalized patients were recruited in an Institutional observational protocol (COVIDBioB Study, Ethical Committee approval no. 34/int/ 2020, ClinicalTrials.gov NCT04318366) and gave written informed consent to compassionate off-label use of anakinra, sarilumab, and tocilizumab. Patients eligible to anti-cytokine therapy were required to have confirmed SARS-CoV-2 infection by reverse-transcriptase polymerase-chain-reaction on nasalpharyngeal swab and radiologically documented bilateral pneumonia. In addition patients were required to have severe COVID-19 as defined by a partial pressure of arterial oxygen/ fraction of inspired oxygen $(\mathrm{PaO} 2 / \mathrm{FiO} 2)$ ratio $\leq 300 \mathrm{mmHg}$ on high flow supplemental oxygen, and a hyper-inflamed phenotype as defined by an elevation of lactate dehydrogenase (LDH) above the upper limit of normal (ULN), and at least one of the following: C-reactive protein (CRP) $\geq 100 \mathrm{mg} / \mathrm{L}$; IL-6 $\geq 40 \mathrm{pg} / \mathrm{ml}$; or ferritin $\geq 900 \mathrm{ng} / \mathrm{ml}$. Patients hospitalized for more than 14 days, on concomitant or previous immunosuppressive agents, or mechanically ventilated were excluded. Patients with uncontrolled systemic infections, total neutrophil count $<1500 /$ $\mathrm{mm} 3$, serum levels of alanine aminotransferase and aspartate aminotransferase more than five times the ULN, diverticulitis/ diverticulosis, and pregnant women were also excluded. Contemporary patients fulfilling the same inclusion/exclusion criteria and matched for age, comorbidities, inflammatory markers, and respiratory parameters were identified and used as a comparison group. These patients did not receive anti-cytokine therapy due to drug unavailability at the time of hospital admission or lack of consent. Patients admitted to intensive care units (ICU) or dying within 24 hours from criteria fulfillment were excluded to avoid potential biases favoring one or the other group.

\section{Study Design and Treatments}

The main objectives of the study were to (i) describe the longterm survival of patients treated with anti-cytokine biologic agents compared to patients treated with local standard of care; (ii) to compare the efficacy of different biologic agents in terms of mortality rate; (iii) to identify possible predictors of response to anti-cytokine therapies. Treatment with biologic drugs was initiated in addition to local standard of care on a compassionate indication within 24 hours from the fulfilment of inclusion criteria outside ICU. Anakinra was administered intravenously at a dose of $5 \mathrm{mg} / \mathrm{Kg}$ twice daily (total daily dose: $10 \mathrm{mg} / \mathrm{kg}$ ) until clinical benefit, defined as sustained improvement of respiratory parameters. Tocilizumab was administered intravenously as a single dose of $400 \mathrm{mg}$, which was repeated after 24 hours if the respiratory function further worsened. Sarilumab was administered intravenously as a single dose of $400 \mathrm{mg}$. All patients received oral therapy with lopinavir/ ritonavir, hydroxychloroquine and a course of azithromycin as per local institutional standard of care at the time of admission (see next section). Supportive therapies with supplemental oxygen and/or non-invasive ventilation (NIV) with continuous positive airway pressure (CPAP, with a positive end expiratory pressure [PEEP] of $10 \mathrm{~cm}$ of $\mathrm{H} 2 \mathrm{O}$ ) were provided at the discretion of the caring clinicians. Patients were prospectively followed-up with daily data collection into an electronic case report form until death or discharge. Patients transferred to rehabilitation were also followed-up through clinical records. There has been no patient or public involvement in the conception, design, and conduction of the present study. 


\section{Standard of Care Treatment}

Antiviral therapy: in the absence of specific contraindications, all patient included in this study received: Hydroxychloroquine 200 mg BD orally and Lopinavir/Ritonavir, 400/100 mg BD orally. Steroids: glucocorticoids were not part of the standard of care and were not used to treat enrolled patients, although in some circumstances they have been used for relieving bronchospasm or for treating hypersensitivity drug reactions. Being on other immunosuppressive agents was considered an exclusion criteria.

TABLE 1 | Clinical and serological features of the patient cohort.

\begin{tabular}{|c|c|c|c|}
\hline & $\begin{array}{c}\text { Biologics } \\
(n=107)\end{array}$ & $\begin{array}{l}\text { Standard of care } \\
\qquad(n=103)\end{array}$ & $\begin{array}{c}p \\
\text { value }\end{array}$ \\
\hline Age (years) & $61(54-72)$ & $62(57-72)$ & 0.15 \\
\hline Male sex, n (\%) & $67(62.6 \%)$ & $57(55 \%)$ & 0.33 \\
\hline \multicolumn{4}{|l|}{ Comorbidities } \\
\hline $\mathrm{HBP}$ & 39 (35\%) & 44 (43\%) & 0.49 \\
\hline CAD & $11(10 \%)$ & 18 (17\%) & 0.17 \\
\hline Type 2 diabetes & $17(16 \%)$ & 21 (20\%) & 0.38 \\
\hline COPD & $4(4 \%)$ & 7 (7\%) & 0.36 \\
\hline Cancer & $5(5 \%)$ & $8(8 \%)$ & 0.58 \\
\hline CRF & $7(7 \%)$ & $7(7 \%)$ & 0.99 \\
\hline $\begin{array}{l}\text { PaO2/FiO2 ratio } \\
\text { (mmHg) } \\
\text { Laboratory values }\end{array}$ & $91(75-132)$ & $105(79-188)$ & 0.1 \\
\hline LAD (125-220 IU/L) & $453(377-579)$ & $474(383-606)$ & 0.38 \\
\hline $\operatorname{CRP}(<6 \mathrm{mg} / \mathrm{L})$ & $154(111-219)$ & $167.6(124.8-231)$ & 0.11 \\
\hline Ferritin (30-400 ng/mL) & $\begin{array}{c}1542(1009- \\
3030)\end{array}$ & $2260(1237-3450)$ & 0.11 \\
\hline IL6 (<7 pg/mL) & $57(37-117)$ & 68.9 (34.65-162) & 0.63 \\
\hline
\end{tabular}

Results are reported as median (interquartile range) unless specified; statistically significant $p$ value $<0.05$. HBP, high blood pressure; CAD, coronary artery disease; COPD, chronic obstructive pulmonary disease; $C R F$, chronic renal failure; $L A D$, lactate dehydrogenase; CRP, C-reactive protein; IL-6, interleukin-6.
Antibiotic therapy: all patients received an initial empiric antibiotic coverage for community acquired/hospital acquired pneumonia based on either daily intravenous Ceftriaxone $2 \mathrm{~g}$ or Azithromycin $500 \mathrm{mg}$. For patients with negative cultures and decreasing inflammatory markers ceftriaxone was discontinued after 6 days of therapy.

\section{Statistical Analysis}

Statistical analysis was performed using Prism software 8.0 (GraphPad Software, La Jolla, CA, USA) and SPSS v26 (SPSS, Chicago, IL-USA). Continuous variables are reported as medians and interquartile ranges. Categorical variables are reported as numbers and percentages. Wilcoxon rank-sum tests were applied to continuous variables and two-tailed Fisher's exact tests were used for categorical variables. Pearson chi-square was employed while analysis frequencies of more than two groups. Survival analysis was performed with the Kaplan-Meier plots, and logrank test was used to compare survival curves. Hazard ratio (HR) of survival were estimated using Cox regression models with backward selection method. Results of the Cox regression model are presented as HR with 95\% confidence interval. Variables with a p-value $<0.2$ on univariate analysis were subsequently entered into the final multivariate model. P-values $<0.05$ were considered statistically significant.

\section{RESULTS}

\section{Baseline Characteristics of the Patients' Cohorts}

210 subjects fulfilling the inclusion criteria were included. 107 $(50.9 \%)$ patients were treated with biologic agents in addition to

TABLE 2 | Clinical and serological features of patients treated with anakinra.

\begin{tabular}{|c|c|c|c|c|c|c|c|c|c|}
\hline & \multirow{2}{*}{$\begin{array}{c}\text { Anakinra } \\
(n=52)\end{array}$} & \multirow{2}{*}{$\begin{array}{l}\text { Standard of care } \\
\qquad(n=88)\end{array}$} & \multirow{2}{*}{$\begin{array}{c}p \\
\text { value }\end{array}$} & \multicolumn{3}{|c|}{$\mathrm{PaO2} / \mathrm{FiO} 2$ ratio $\geq 100 \mathrm{mmHg}$} & \multicolumn{3}{|c|}{$\mathrm{PaO} 2 / \mathrm{FiO} 2$ ratio $<100 \mathrm{mmHg}$} \\
\hline & & & & $\begin{array}{c}\text { Anakinra } \\
(n=16)\end{array}$ & $\begin{array}{l}\text { Standard of care } \\
\qquad(n=43)\end{array}$ & $\begin{array}{c}\mathrm{p} \\
\text { value }\end{array}$ & $\begin{array}{c}\text { Anakinra } \\
(n=36)\end{array}$ & $\begin{array}{l}\text { Standard of care } \\
\qquad(n=45)\end{array}$ & $\begin{array}{c}\mathbf{p} \\
\text { value }\end{array}$ \\
\hline Age (years) & $63(55-74)$ & $62(57-73)$ & & $60(51-71)$ & $61(55-72)$ & 0.77 & $64(56-74)$ & $63(58-73)$ & 0.9 \\
\hline Male sex, $\mathrm{n}(\%)$ & $33(63 \%)$ & $50(57 \%)$ & 0.59 & $13(81 \%)$ & $31(72 \%)$ & 0.74 & $20(56 \%)$ & $19(42 \%)$ & 0.27 \\
\hline \multicolumn{10}{|l|}{ Comorbidities } \\
\hline $\mathrm{HBP}$ & 22 (42\%) & 39 (44\%) & 0.86 & $4(25 \%)$ & 17 (40\%) & 0.37 & $18(50 \%)$ & $22(49 \%)$ & 0.99 \\
\hline CAD & $6(11 \%)$ & $16(18 \%)$ & 0.34 & $0(0 \%)$ & $8(19 \%)$ & 0.09 & $6(16 \%)$ & $8(18 \%)$ & 0.99 \\
\hline Type 2 diabetes & $12(23 \%)$ & $18(21 \%)$ & 0.83 & $1(6 \%)$ & $8(19 \%)$ & 0.42 & $11(30 \%)$ & $10(22 \%)$ & 0.45 \\
\hline COPD & $3(6 \%)$ & $7(8 \%)$ & 0.74 & $0(0 \%)$ & $3(7 \%)$ & 0.56 & 3 (8\%) & 4 (9\%) & 0.99 \\
\hline Cancer & $2(4 \%)$ & $8(9 \%)$ & 0.32 & 1 (6\%) & 7 (16\%) & 0.43 & 1 (3\%) & $1(2 \%)$ & 0.99 \\
\hline CRF & $4(8 \%)$ & $4(5 \%)$ & 0.47 & $0(0 \%)$ & $1(2 \%)$ & 0.99 & $4(11 \%)$ & $3(7 \%)$ & 0.69 \\
\hline $\begin{array}{l}\text { PaO2/FiO2 ratio } \\
\text { (mmHg) } \\
\text { Laboratory values }\end{array}$ & $81(70-113)$ & $96(72-143)$ & 0.09 & $143(117-181)$ & 143 (122-189) & 0.84 & 77 (67-85) & $73(61-86)$ & 0.74 \\
\hline $\begin{array}{l}\text { LAD } \\
(125-220 \mathrm{IU} / \mathrm{L})\end{array}$ & $458(372-580)$ & $510(393-630)$ & 0.21 & $392(286-551)$ & $428(383-542)$ & 0.25 & $499(389-591)$ & $565(436-726)$ & 0.08 \\
\hline $\mathrm{CRP}(<6 \mathrm{mg} / \mathrm{L})$ & 157 (120-224) & $171(122-232)$ & 0.36 & $130(102-162)$ & $153(119-199)$ & 0.12 & $165(128-240)$ & 202 (135-238. & 0.65 \\
\hline $\begin{array}{l}\text { Ferritin } \\
(30-400 \mathrm{ng} / \mathrm{mL})\end{array}$ & $\begin{array}{c}1547(937- \\
3521)\end{array}$ & $2347(1242-3549)$ & 0.17 & $\begin{array}{c}1773(912- \\
3347)\end{array}$ & $2486(1291-3436)$ & 0.4 & $\begin{array}{c}1462(929- \\
3847)\end{array}$ & $1717(1198-3871)$ & 0.33 \\
\hline IL6 (<7 pg/mL) & 48 (27-99) & $65(37-172)$ & 0.63 & 75 (69-82) & 47 (26-153) & 0.22 & $59(42-831)$ & 105 (66-287) & 0.41 \\
\hline
\end{tabular}

Results are reported as median (interquartile range) unless specified; statistically significant $p$ value $<0.05$. HBP, high blood pressure; CAD, coronary artery disease; COPD, chronic obstructive pulmonary disease; CRF, chronic renal failure; LAD, lactate dehydrogenase; CRP, C-reactive protein; IL-6, interleukin-6. 
TABLE 3 | Clinical and serological features of patients treated with sarilumab.

\begin{tabular}{|c|c|c|c|c|c|c|c|c|c|}
\hline & \multirow{2}{*}{$\begin{array}{l}\text { Sarilumab } \\
\qquad(n=25)\end{array}$} & \multirow{2}{*}{$\begin{array}{l}\text { Standard of care } \\
\qquad(n=68)\end{array}$} & \multirow{2}{*}{$\begin{array}{c}p \\
\text { value }\end{array}$} & \multicolumn{3}{|c|}{$\mathrm{PaO} 2 /$ FiO2 ratio $\geq 100 \mathrm{mmHg}$} & \multicolumn{3}{|c|}{$\mathrm{PaO} 2 / \mathrm{FiO} 2$ ratio $<100 \mathrm{mmHg}$} \\
\hline & & & & $\begin{array}{l}\text { Sarilumab } \\
\qquad(n=8)\end{array}$ & $\begin{array}{l}\text { Standard of care } \\
\qquad(n=33)\end{array}$ & $\begin{array}{c}\mathbf{p} \\
\text { value }\end{array}$ & $\begin{array}{l}\text { Sarilumab } \\
\qquad(n=17)\end{array}$ & $\begin{array}{l}\text { Standard of care } \\
\qquad(n=35)\end{array}$ & $\begin{array}{c}p \\
\text { value }\end{array}$ \\
\hline Age (years) & $56(50-62)$ & $61(55-67)$ & 0.07 & $56(52-62)$ & $60(52-64)$ & 0.74 & $56(48-60)$ & $61(57-64)$ & 0.07 \\
\hline Male sex, n (\%) & $16(64 \%)$ & 37 (54\%) & 0.48 & $6(75 \%)$ & 23 (70\%) & 0.99 & $10(59 \%)$ & $14(40 \%)$ & 0.24 \\
\hline \multicolumn{10}{|l|}{ Comorbidities } \\
\hline $\mathrm{HBP}$ & $5(20 \%)$ & 27 (39\%) & 0.09 & $1(12 \%)$ & 10 (30\%) & 0.41 & $4(23 \%)$ & 17 (49\%) & 0.13 \\
\hline CAD & $2(8 \%)$ & $8(12 \%)$ & 0.72 & $1(12 \%)$ & $2(6 \%)$ & 0.49 & $1(6 \%)$ & $6(17 \%)$ & 0.4 \\
\hline Type 2 diabetes & $1(4 \%)$ & $14(21 \%)$ & 0.07 & $0(0 \%)$ & $6(18 \%)$ & 0.32 & $1(6 \%)$ & $8(23 \%)$ & 0.24 \\
\hline COPD & $0(0 \%)$ & 5 (7\%) & 0.32 & $0(0 \%)$ & $1(3 \%)$ & 0.99 & 0 (0\%) & $4(11 \%)$ & 0.29 \\
\hline Cancer & $1(4 \%)$ & $6(9 \%)$ & 0.67 & $0(0 \%)$ & $6(18 \%)$ & 0.32 & $1(6 \%)$ & $0(0 \%)$ & 0.33 \\
\hline CRF & $0(0 \%)$ & 2 (3\%) & 0.9 & $0(0 \%)$ & $0(0 \%)$ & 0.99 & $0(0 \%)$ & $2(6 \%)$ & 0.99 \\
\hline $\begin{array}{l}\text { PaO2/FiO2 ratio } \\
\text { (mmHg) } \\
\text { Laboratory values }\end{array}$ & $87(74-123)$ & $96(71-146)$ & 0.38 & $138(122-184)$ & $148(116-187)$ & 0.98 & $81(69-87)$ & $72(61-89)$ & 0.48 \\
\hline $\begin{array}{l}\text { LAD } \\
(125-220 \mathrm{IU} / \mathrm{L})\end{array}$ & $477(420-591)$ & $522(403-632)$ & 0.47 & $444(327-489)$ & $459(386-541)$ & 0.46 & $488(438-602)$ & $585(447-726)$ & 0.21 \\
\hline $\mathrm{CRP}(<6 \mathrm{mg} / \mathrm{L})$ & $132(102-219)$ & $176(122-230)$ & 0.23 & $143(112-179)$ & $153(118-192)$ & 0.58 & $122(100-241)$ & $202(132-238)$ & 0.24 \\
\hline $\begin{array}{l}\text { Ferritin } \\
(30-400 \mathrm{ng} / \mathrm{mL})\end{array}$ & $\begin{array}{c}1890(1028- \\
3050)\end{array}$ & $2486(1237-3500)$ & 0.36 & $\begin{array}{c}1098(876- \\
1550)\end{array}$ & $1876(1217-3450)$ & 0.07 & $\begin{array}{c}2611(1410- \\
3883)\end{array}$ & $1482(1077-3720)$ & 0.7 \\
\hline IL6 (<7 pg/mL) & $60(38-129)$ & $51(28-143)$ & 0.41 & $88(43-164)$ & $42(26-170)$ & 0.27 & $60(36-127)$ & 99 (66-270) & 0.1 \\
\hline
\end{tabular}

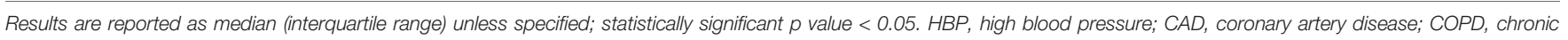
obstructive pulmonary disease; CRF, chronic renal failure; LAD, lactate dehydrogenase; CRP, C-reactive protein; IL-6, interleukin-6.

TABLE 4 | Clinical and serological features of patients treated with tocilizumab.

\begin{tabular}{|c|c|c|c|c|c|c|c|c|c|}
\hline & \multirow{2}{*}{$\begin{array}{l}\text { Tocilizumab } \\
(n=30)\end{array}$} & \multirow{2}{*}{$\begin{array}{l}\text { Standard of care } \\
\qquad(n=103)\end{array}$} & \multirow{2}{*}{$\begin{array}{c}p \\
\text { value }\end{array}$} & \multicolumn{3}{|c|}{$\mathrm{PaO2} / \mathrm{FiO} 2$ ratio $\geq 100 \mathrm{mmHg}$} & \multicolumn{3}{|c|}{$\mathrm{PaO} 2 / \mathrm{FiO} 2$ ratio $<100 \mathrm{mmHg}$} \\
\hline & & & & $\begin{array}{c}\text { Tocilizumab } \\
(n=18)\end{array}$ & $\begin{array}{l}\text { Standard of care } \\
\qquad(n=59)\end{array}$ & $\begin{array}{c}p \\
\text { value }\end{array}$ & $\begin{array}{c}\text { Tocilizumab } \\
(n=12)\end{array}$ & $\begin{array}{l}\text { Standard of care } \\
\qquad(n=44)\end{array}$ & $\begin{array}{c}p \\
\text { value }\end{array}$ \\
\hline Age (years) & $62.5(53-74)$ & $62(57-72)$ & 0.86 & $58(51-73)$ & $61(55-71)$ & 0.56 & $72(59-74)$ & $63(58-73)$ & 0.56 \\
\hline Male sex, n (\%) & $18(60 \%)$ & 57 (55\%) & 0.68 & $12(67 \%)$ & 38 (64\%) & 0.99 & $6(50 \%)$ & 19 (43\%) & 0.75 \\
\hline \multicolumn{10}{|l|}{ Comorbidities } \\
\hline $\mathrm{HBP}$ & 12 (40\%) & 44 (43\%) & 0.83 & 7 (39\%) & 22 (37\%) & 0.99 & 5 (42\%) & 23 (52\%) & 0.75 \\
\hline CAD & $3(10 \%)$ & 18 (17\%) & 0.4 & $0(0 \%)$ & 10 (17\%) & 0.1 & $3(25 \%)$ & $8(18 \%)$ & 0.69 \\
\hline Type 2 diabetes & $4(13 \%)$ & $21(20 \%)$ & 0.44 & $2(11 \%)$ & 10 (17\%) & 0.72 & $2(17 \%)$ & $11(25 \%)$ & 0.71 \\
\hline COPD & 1 (3\%) & 7 (7\%) & 0.68 & $0(0 \%)$ & $3(5 \%)$ & 0.99 & 1 (8\%) & 4 (9\%) & 0.99 \\
\hline Cancer & $2(6 \%)$ & $8(8 \%)$ & 0.99 & $1(5 \%)$ & 7 (12\%) & 0.67 & 1 (8\%) & $1(2 \%)$ & 0.39 \\
\hline CRF & $3(10 \%)$ & 7 (7\%) & 0.69 & $0(0 \%)$ & $3(5 \%)$ & 0.99 & $3(25 \%)$ & $4(9 \%)$ & 0.16 \\
\hline $\begin{array}{l}\mathrm{PaO} 2 / \mathrm{FiO} 2 \text { ratio } \\
(\mathrm{mmHg})\end{array}$ & $111(84-181)$ & $121(91-221)$ & 0.83 & $176(117-187)$ & $172(127-229)$ & 0.35 & $81(70-86)$ & $73(61-86)$ & 0.26 \\
\hline \multicolumn{10}{|l|}{ Laboratory values } \\
\hline $\begin{array}{l}\text { LAD } \\
(125-220 \mathrm{IU} / L)\end{array}$ & $441(358-552)$ & $474(383-606)$ & 0.39 & $443(354-552)$ & $415(377-540)$ & 0.66 & $438(362-583)$ & $565(436-726)$ & 0.08 \\
\hline $\mathrm{CRP}(<6 \mathrm{mg} / \mathrm{L})$ & $143(92-212)$ & $167.6(124-231)$ & 0.17 & $124(68-187)$ & $153(123-192)$ & 0.09 & $186(139-234)$ & $202.6(135-238)$ & 0.77 \\
\hline $\begin{array}{l}\text { Ferritin } \\
(30-400 \mathrm{ng} / \mathrm{mL})\end{array}$ & $\begin{array}{c}1400(1054- \\
2654)\end{array}$ & 2260 (1237-3450) & 0.1 & $\begin{array}{c}1433(1104- \\
3079)\end{array}$ & 2326 (1254-3215) & 0.47 & $\begin{array}{c}1228(789- \\
2573)\end{array}$ & 1717 (1198-3871) & 0.07 \\
\hline IL6 (<7 pg/mL) & $69(50-456)$ & $68.9(34-162)$ & 0.73 & 56 (29-229) & $51(26-116)$ & 0.71 & $45(24-52)$ & 105 (66-286) & 0.11 \\
\hline
\end{tabular}

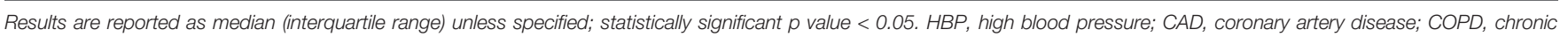
obstructive pulmonary disease; $C R F$, chronic renal failure; LAD, lactate dehydrogenase; CRP, C-reactive protein; IL-6, interleukin-6.

local standard of care: 52 (48.6\%) with anakinra; 30 (28\%) with tocilizumab; and 25 (23.4\%) with sarilumab (Tables 1-4). 103 (49.1\%) concomitantly hospitalized patients matched according to the inclusion criteria and treated with local standard of care were used as comparators (Table 1). All patients were on highflow oxygen supplementation or on NIV as per clinical judgement due to acute respiratory distress syndrome (ARDS) $(\mathrm{PaO} 2 / \mathrm{FiO} 2$ ratio $<300$ with a $\mathrm{PEEP} \geq 5 \mathrm{~cm} \mathrm{H} 2 \mathrm{O})$.

\section{Predictors of Survival}

After a median follow-up time of 111 days (range 3-186) from the fulfilment of inclusion criteria, 21 (19.6\%) patients treated with biologics and $36(34.9 \%)$ patients treated with standard of care died $(p=0.01)$. Treatment with biologic drugs was associated with a lower mortality risk compared to patients treated with standard of care (Hazard Ratio (HR) 0.48; 95\% CI $0.29-0.81 ; p=0.006$ ) (Figure 1A). When the efficacy of each 

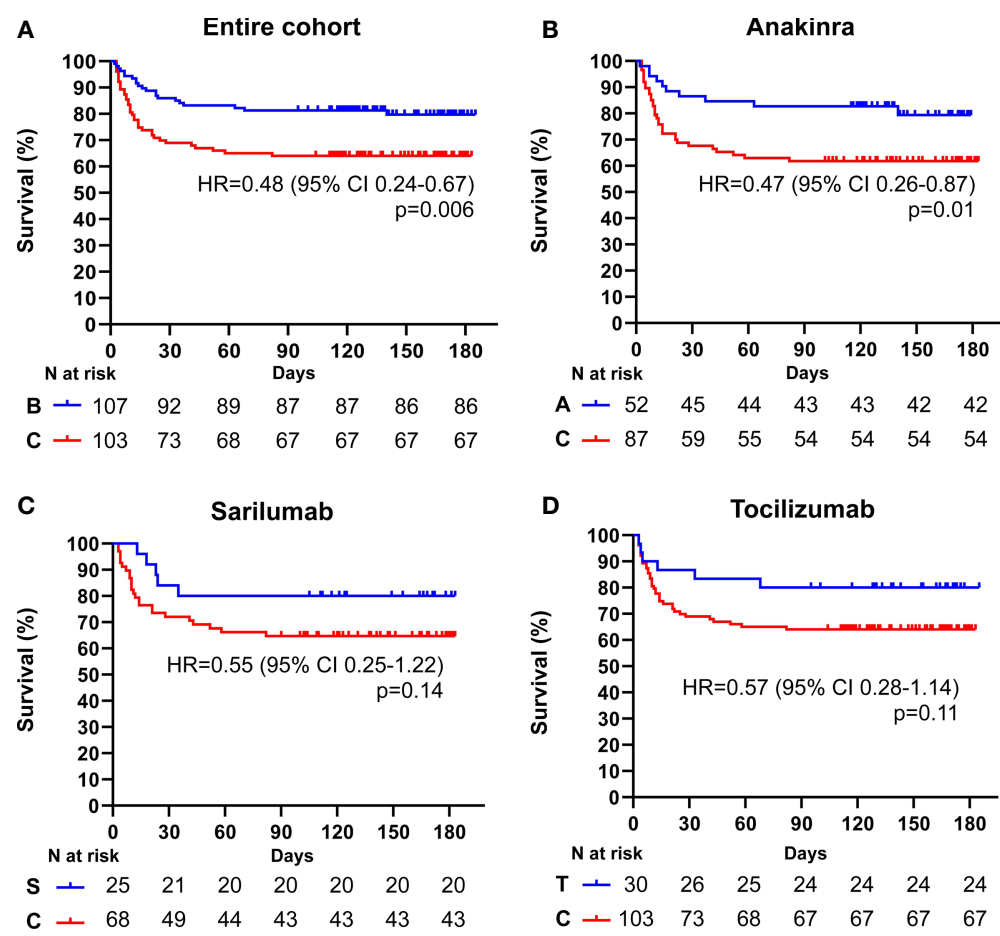

FIGURE 1 | Cumulative incidence of long-term survival of patients treated with biologic drugs compared to patients treated with standard of care: entire patient cohort. Kaplan-Meier curves showing the cumulative incidence of overall survival up to 186 days of the entire cohort of patients treated with biologic drugs (A), anakinra (B), sarilumab (C), and tocilizumab (D) compared to patients treated with standard of care. Time to death is expressed in days from the day of enrolment. A, anakinra; B, biologic drugs; C, comparators treated with standard of care; S, sarilumab; T, tocilizumab; HR, hazard ratio; Cl, confidence interval. Statistically significant $p$ value $<0.05$

biologic agent was compared to matched controls treated with standard of care, anakinra (HR 0.47; 95\% CI 0.26-0.87; $\mathrm{p}=0.01$ ), but not sarilumab (HR 0.55; 95\% CI $0.25-1.22 ; \mathrm{p}=0.14)$ nor

TABLE 5 | Baseline univariate and multivariate predictors of mortality in the patients' cohort.

\begin{tabular}{lccccc}
\hline & \multicolumn{2}{c}{ Univariate analysis } & & \multicolumn{2}{c}{ Multivariate analysis } \\
\cline { 2 - 3 } \cline { 5 - 6 } & HR (95\% Cl) & $\begin{array}{c}\mathbf{p} \\
\text { value }\end{array}$ & & HR (95\% Cl) & $\begin{array}{c}\mathbf{p} \\
\text { value }\end{array}$ \\
\hline Age & $1.05(1.02-1.08)$ & $\mathbf{0 . 0 0 0 1}$ & & $1.05(1.02-1.08)$ & $\mathbf{0 . 0 0 1}$ \\
Sex (F) & $0.64(0.37-1.1)$ & 0.11 & & & \\
HBP & $1.62(0.97-2.71)$ & 0.07 & & $1.39(0.77-2.51)$ & 0.17 \\
CAD & $2.23(1.2-4.16)$ & $\mathbf{0 . 0 1 1}$ & & $1.59(0.82-2.99)$ & 0.16 \\
PaO2/FiO2 & $0.997(0.99-1.00)$ & 0.09 & & $0.998(0.99-1.002)$ & 0.51 \\
ratio (mmHg) & & & & \\
LAD & $1.002(1.001-1.003)$ & $\mathbf{0 . 0 0 2}$ & & $1.002(1-1.003)$ & $\mathbf{0 . 0 0 3}$ \\
CRP & $1.003(1-1.01)$ & $\mathbf{0 . 0 5}$ & & $1.001(0.99-1.01)$ & 0.36 \\
Ferritin & $1(1-1)$ & 0.56 & & \\
IL-6 & $1(1-1)$ & 0.11 & & \\
Treatment with & $0.4(0.23-0.68)$ & $\mathbf{0 . 0 0 1}$ & & $0.49(0.27-0.87)$ & $\mathbf{0 . 0 1}$ \\
biologics & & & & & \\
\hline
\end{tabular}

Results are reported as hazard ratio and $95 \%$ confidence interval; statistically significant $p$ value < 0.05. HBP, high blood pressure; $C A D$, coronary artery disease; $L A D$, lactate dehydrogenase; CRP, C-reactive protein; IL-6, interleukin-6; $\mathrm{HR}$, hazard ratio; $\mathrm{Cl}$, confidence interval.

Bold values indicate that the $p$ values are statistically significant. tocilizumab (HR 0.57; 95\% CI 0.28-1.14; $\mathrm{p}=0.11$ ), provided a statistically significant lower mortality risk (Figures 1B-D and Tables 2-4). In the multivariate analysis, treatment with cytokine blocking agents was associated with improved patient survival while older age and high concentration of lactate

TABLE 6 | Baseline univariate and multivariate predictors of mortality in patients treated with biologic drugs.

\begin{tabular}{lccccc}
\hline & \multicolumn{2}{c}{ Univariate analysis } & & \multicolumn{2}{c}{ Multivariate analysis } \\
\cline { 2 - 3 } \cline { 5 - 6 } & HR (95\% Cl) & $\begin{array}{c}\mathbf{p} \\
\text { value }\end{array}$ & & HR (95\% Cl) & $\begin{array}{c}\mathbf{p} \\
\text { value }\end{array}$ \\
\hline Age & $1.04(0.99-1.08)$ & 0.07 & & $1.043(1-1.09)$ & $\mathbf{0 . 0 4 8}$ \\
Sex (F) & $1.039(0.306-3.527)$ & 0.95 & & \\
HBP & $1.28(0.54-3.04)$ & 0.574 & & \\
CAD & $4.89(1.89-12.64)$ & $\mathbf{0 . 0 0 1}$ & & $1.95(0.61-6.3)$ & 0.27 \\
PaO2/FiO2 ratio & $0.98(0.96-99)$ & $\mathbf{0 . 0 1}$ & & $0.98(0.965-0.997)$ & $\mathbf{0 . 0 4 5}$ \\
(mmHg) & & & & \\
LAD & $1.004(1.00-1.01)$ & $\mathbf{0 . 0 0 2}$ & & $1.004(1.001-1.007)$ & $\mathbf{0 . 0 1}$ \\
CRP & $1.001(0.996-1.007)$ & 0.61 & & \\
Ferritin & $1(1-1)$ & 0.65 & & \\
IL-6 & $1(0.99-1.01)$ & 0.12 & & \\
\hline
\end{tabular}

Results are reported as hazard ratio and $95 \%$ confidence interval; statistically significant $p$ value < 0.05. $H B P$, high blood pressure; $C A D$, coronary artery disease; $L A D$, lactate dehydrogenase; CRP, C-reactive protein; IL-6, interleukin-6; HR, hazard ratio; Cl, confidence interval.

Bold values indicate that the $p$ values are statistically significant. 
TABLE 7 | Clinical and serological features of patients with a PaO2/FiO2 ratio $<100 \mathrm{mmHg}$ and $\geq 100 \mathrm{mmHg}$.

\begin{tabular}{|c|c|c|c|c|c|c|}
\hline & \multicolumn{3}{|c|}{$\mathrm{PaO} 2 / \mathrm{FiO} 2$ ratio $\geq 100 \mathrm{mmHg}$} & \multicolumn{3}{|c|}{$\mathrm{PaO2} /$ FiO2 ratio $<100 \mathrm{mmHg}$} \\
\hline & Biologics $(n=42)$ & Standard of care $(n=59)$ & $p$ value & Biologics $(n=65)$ & Standard of care $(n=44)$ & $p$ value \\
\hline Age (years) & $58(51-68.25)$ & $61(55-71)$ & 0.46 & $62(55.5-73.5)$ & $63(58-73)$ & 0.27 \\
\hline Male sex, n (\%) & 31 (74\%) & 38 (64\%) & 0.51 & 36 (55\%) & 19 (43\%) & 0.22 \\
\hline \multicolumn{7}{|l|}{ Comorbidities } \\
\hline HBP & $12(29 \%)$ & $22(37 \%)$ & 0.39 & 27 (42\%) & $23(52 \%)$ & 0.33 \\
\hline CAD & $1(2 \%)$ & $10(17 \%)$ & 0.03 & $10(15 \%)$ & $8(18 \%)$ & 0.99 \\
\hline Type 2 diabetes & $3(7 \%)$ & $10(17 \%)$ & 0.23 & $14(22 \%)$ & $11(25 \%)$ & 0.65 \\
\hline COPD & $0(0 \%)$ & $3(5 \%)$ & 0.25 & $4(6 \%)$ & $4(9 \%)$ & 0.71 \\
\hline Cancer & $2(5 \%)$ & $7(12 \%)$ & 0.3 & $3(5 \%)$ & $1(2 \%)$ & 0.65 \\
\hline CRF & $0(0 \%)$ & $3(5 \%)$ & 0.26 & $7(11 \%)$ & $4(9 \%)$ & 0.99 \\
\hline $\mathrm{PaO} / \mathrm{FiO} 2$ ratio $(\mathrm{mmHg})$ & $144(119-185)$ & $172(127-229)$ & 0.1 & $77(67-87)$ & $73(61-86)$ & 0.59 \\
\hline \multicolumn{7}{|l|}{ Laboratory values } \\
\hline LAD (125-220 IU/L) & $432(350-547)$ & 415 (377-540) & 0.71 & 487 (407-595) & 565 (436-726) & 0.07 \\
\hline $\mathrm{CRP}(<6$ mg/L) & $134(97-172)$ & 153 (123-192) & 0.07 & 166 (122-239) & $202(135-238)$ & 0.41 \\
\hline Ferritin $(30-400 \mathrm{ng} / \mathrm{mL})$ & $1422(1054-2835)$ & 2326 (1254-3215) & 0.18 & 1731 (938-3099) & $1717(1198-3871)$ & 0.28 \\
\hline IL-6 (< 7 pg/mL) & $56(37-113)$ & $51(26-116)$ & 0.49 & $58(37-120)$ & 105 (66-286) & 0.08 \\
\hline
\end{tabular}

Results are reported as median (interquartile range) unless specified; statistically significant $p$ value $<0.05$. HBP, high blood pressure; CAD, coronary artery disease; COPD, chronic obstructive pulmonary disease; CRF, chronic renal failure; LAD, lactate dehydrogenase; CRP, C-reactive protein; IL-6, interleukin-6.

Bold values indicate that the $p$ values are statistically significant.

A

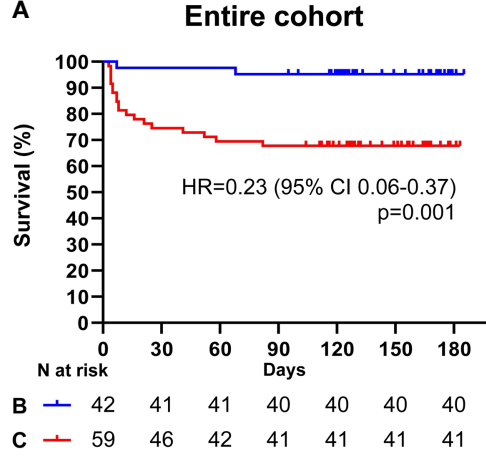

C

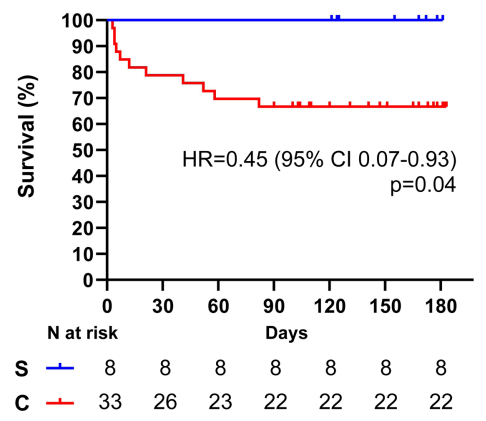

B

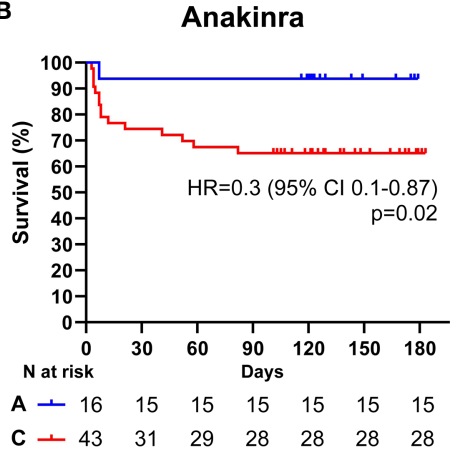

D

Tocilizumab



FIGURE 2 | Cumulative incidence of long-term survival of patients treated with biologic drugs compared to patients treated with standard of care (PaO2/FiO2 ratio $\geq$ $\mathrm{mmHg}$ ). Kaplan-Meier curves showing the cumulative incidence of overall survival up to 186 days of patients presenting with a $\mathrm{PaO} 2 / \mathrm{FiO} 2$ ratio $\geq \mathrm{mmHg}$ and treated with biologic drugs (A), anakinra (B), sarilumab (C), and tocilizumab (D), compared to standard of care. Time to death is expressed in days from the day of enrolment. A, anakinra; B, biologic drugs; C, comparators treated with standard of care; S, sarilumab; T, tocilizumab; HR, hazard ratio; Cl, confidence interval. Statistically significant $p$ value $<0.05$. 
dehydrogenase were independently associated with overall increased mortality (Table 5). When considering only patients treated with biologic therapies, COVID-19 related mortality was independently associated with older age, high concentration of lactate dehydrogenase, and low $\mathrm{PaO} 2 / \mathrm{FiO}_{2}$ ratio at the time of drug infusion (Table 6).

\section{Impact of Respiratory Distress on Clinical Response to Biologic Therapy}

Because $\mathrm{PaO} 2 / \mathrm{FiO} 2$ ratio represented an independent predictor of COVID-19 related mortality at multivariate analysis in patients treated with biologic agents, as well as a surrogate of disease progression and lung consolidation (4), we assessed the efficacy of anti-cytokine therapies on long-term survival in patients with moderate $(\mathrm{PaO} 2 / \mathrm{FiO} 2$ ratio $\geq 100 \mathrm{mmHg})$ and severe $(\mathrm{PaO} 2 / \mathrm{FiO} 2$ ratio $<100 \mathrm{mmHg})$ ARDS.

Among the 101 subjects with a $\mathrm{PaO} 2 / \mathrm{FiO} 2$ ratio $\geq 100$ $\mathrm{mmHg}, 42$ (41.6\%) received an IL-inhibitor and 59 (54\%) were treated with standard of care. The two populations displayed similar epidemiological, clinical, and inflammatory parameters (Table 7). After a mean follow-up time of 113 days (range 95186), 2 (4.7\%) patients treated with biologic agents and 18 $(30.5 \%)$ patients treated with standard of care died $(\mathrm{p}<0.001)$ (HR 0.23; 95\% CI 0.1-0.55; $\mathrm{p}=0.001$ ) (Figure 2A). At multivariate analysis ferritin elevation and treatment with

TABLE 8 | Baseline univariate and multivariate predictors of mortality in patients with a $\mathrm{PaO} 2 / \mathrm{FiO} 2$ ratio $<100 \mathrm{mmHg}$ and $\geq 100 \mathrm{mmHg}$.

\begin{tabular}{|c|c|c|c|c|}
\hline & HR (95\% Cl) & $p$ value & HR (95\% Cl) & $p$ value \\
\hline \multicolumn{5}{|l|}{$\begin{array}{l}\mathrm{PaO2} / \mathrm{FiO} 2 \\
\text { ratio }\end{array}$} \\
\hline \multicolumn{5}{|l|}{$\geq 100 \mathrm{mmHg}$} \\
\hline Age & $1.05(1-1.1)$ & 0.038 & $1.05(0.98-1.13)$ & 0.16 \\
\hline $\operatorname{Sex}(F)$ & $0.56(0.23-1.39)$ & 0.21 & & \\
\hline HBP & $2.32(0.99-5.48)$ & 0.07 & $1.84(0.35-9.67)$ & 0.47 \\
\hline CAD & $1.73(0.5-5.96)$ & 0.39 & & \\
\hline LAD & $1.001(1-1.003)$ & 0.14 & & \\
\hline CRP & $1.006(1-1.01)$ & 0.04 & $1.004(0.99-1.01)$ & 0.22 \\
\hline IL-6 & $1(1-1)$ & 0.14 & & \\
\hline Ferritin & $1(1-1)$ & 0.008 & $1(1-1)$ & 0.01 \\
\hline $\begin{array}{l}\text { Treatment } \\
\text { with biologics }\end{array}$ & $0.1(0.02-0.42)$ & 0.002 & $0.09(0.02-0.51)$ & 0.006 \\
\hline $\begin{array}{l}\mathrm{PaO} 2 / \mathrm{FiO} 2 \\
\text { ratio }\end{array}$ & \multicolumn{3}{|c|}{ ratio } & \\
\hline \multicolumn{5}{|l|}{$<100 \mathrm{mmHg}$} \\
\hline Age & $1.05(1.02-1.09)$ & 0.004 & $1.054(1.02-1.09)$ & 0.003 \\
\hline $\operatorname{Sex}(F)$ & $0.7(0.36-1.35)$ & 0.29 & & \\
\hline HBP & $1.21(0.63-2.3)$ & 0.57 & & \\
\hline CAD & $2.37(1.15-4.91)$ & 0.02 & $1.63(0.77-3.45)$ & 0.2 \\
\hline LAD & $1.002(1-1.003)$ & 0.02 & $1.002(1-1.003)$ & 0.013 \\
\hline CRP & $1.001(0.99-1.01)$ & 0.57 & & \\
\hline IL-6 & $1(1-1)$ & 0.37 & & \\
\hline Ferritin & $1(1-1)$ & 0.39 & & \\
\hline $\begin{array}{l}\text { Treatment } \\
\text { with biologics }\end{array}$ & $0.55(0.28-1.04)$ & 0.07 & $0.66(0.33-1.33)$ & 0.24 \\
\hline
\end{tabular}

Results are reported as hazard ratio and 95\% confidence interval; statistically significant $p$ value < 0.05. $H B P$, high blood pressure; $C A D$, coronary artery disease; $L A D$, lactate dehydrogenase; CRP, C-reactive protein; IL-6, interleukin-6; HR, hazard ratio; Cl, confidence interval.

Bold values indicate that the $p$ values are statistically significant. standard of care represented independent predictors of death while treatment with anti-cytokine therapy represented a protective factor (Table 8). Anakinra, sarilumab, and tocilizumab were each associated with a statistically significant survival advantage compared to matched comparators $(\mathrm{p}<0.05$ for all comparisons) (Figures 2B-D and Tables 2-4).

Among the 109 patients with a $\mathrm{PaO} 2 / \mathrm{FiO} 2$ ratio $<100$ $\mathrm{mmHg}, 65$ (59.6\%) received an IL-inhibitor and 44 (40.4\%) were treated with standard of care. The two populations displayed similar epidemiological, clinical, and inflammatory parameters (Table 7). After a mean follow-up time of 110 days (range 95-170), 19 (29.2\%) patients treated with biologic agents and $18(40.9 \%)$ patients treated with only local standard of care died $(\mathrm{p}=0.21)(\mathrm{HR} 0.61 ; 95 \%$ CI 0.31-1.19; $\mathrm{p}=0.15)$ (Figure 3A). At multivariate analysis, lactate dehydrogenase and age represented independent risk factors for death (Table 8). In this cohort of critical patients, treatment with anakinra but not sarilumab nor tocilizumab was associated with a statistically significant lower mortality risk compared to controls (HR 0.46; 95\% CI 0.22-0.94; $\mathrm{p}=0.04$ ) (Figures 3B-D and Tables 2-4).

\section{DISCUSSION}

During the first COVID-19 pandemic wave that struck Northern Italy between February and May 2020, our Hospital was at the frontline of an unprecedented health emergency $(13,14)$. Based on accumulating evidence about a maladaptive inflammatory response to Sars-CoV-2 in severe COVID-19 cases, we developed a set of stringent criteria to identify hyper-inflamed COVID-19 patients at risk of rapid deterioration who might have benefitted from cytokine-blocking therapies $(3,4,9,10,15,16)$. In the present study, all patients treated with anakinra, sarilumab, or tocilizumab were retrospectively matched according to respiratory and inflammatory parameters, and compared to a cohort of contemporary patients featuring similar characteristics treated with standard of care alone.

By analyzing clinical and serological variables associated with positive response to anti-cytokine therapies, we found that IL-1 and IL-6 inhibition improved long-term survival when initiated in the early phases of COVID-19 pneumonia before the establishment of severe ARDS (namely, $\mathrm{PaO} 2 / \mathrm{FiO} 2$ ratio $<100$ $\mathrm{mmHg}$ ). The survival advantage offered by sarilumab and tocilizumab, in fact, was lost in patients with severe respiratory failure, and the efficacy of anakinra was markedly reduced although still proving superior to standard management. These findings demonstrate that the likelihood of response to IL-1 or IL- 6 blocking strategies in patients with COVID-19 depends on the degree of respiratory impairment at the time of treatment administration and underscore the existence of a window of opportunity in which cytokine-blocking agents - as well as of more common anti-inflammatory therapies such as glucocorticoids and colchicine - might effectively counteract rampant inflammation in COVID-19 (17-19). In addition, our observations suggest that IL- 6 is probably not the only driving pathway of severe COVID-19 and that molecules upstream of IL-6 in the inflammatory cascade, such as IL-1, might represent 


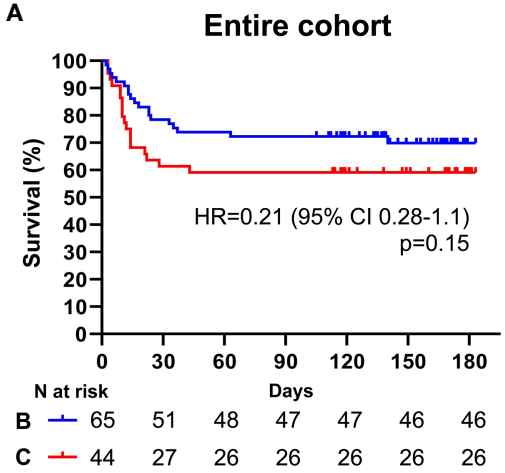

C

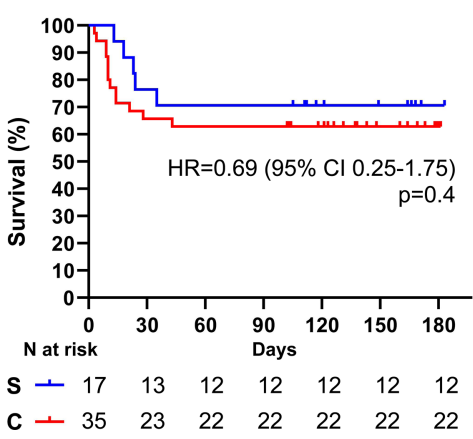

B

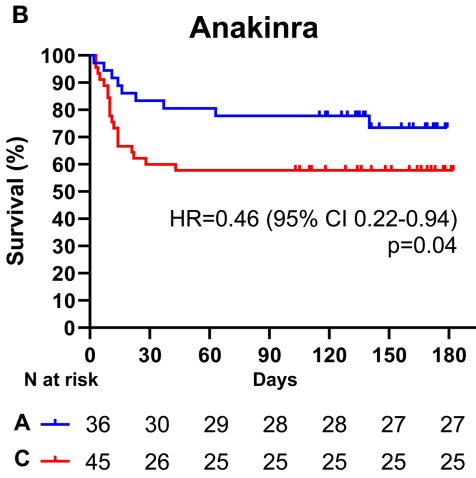

D

Tocilizumab



FIGURE 3 | Cumulative incidence of long-term survival of patients treated with biologic drugs compared to patients treated with standard of care (PaO2/FiO2 ratio < $\mathrm{mmHg}$ ). Kaplan-Meier curves showing the cumulative incidence of overall survival up to 186 days of patients presenting with a $\mathrm{PaO} 2 / \mathrm{FiO} 2 \mathrm{ratio}<\mathrm{mmHg}$ and treated with biologic drugs (A), anakinra (B), sarilumab (C), and tocilizumab (D), compared to standard of care. Time to death is expressed in days from the day of enrolment. A, anakinra; B, biologic drugs; C, comparators treated with standard of care; S, sarilumab; T, tocilizumab; HR, hazard ratio; Cl, confidence interval. Statistically significant $\mathrm{p}$ value $<0.05$.

more suitable targets to quench Sars-CoV-2 induced hyperinflammation. These results expand on our previous experience about the higher efficacy of IL-1 and IL-6 blockade in COVID-19 patients with elevated C-reactive protein and indicate that anti-cytokine treatments should be tailored not only on the inflammatory phenotype but also on the respiratory status in order to implement the selection of patients most likely to benefit from cytokine-targeted therapies $(3,20)$.

This work has major strengths and also limitations. In particular, results were obtained on a large and homogeneous cohort of patients that was enrolled with uniform stringent criteria and managed in the same referral center. In addition, this cohort was assembled before the reports of glucocorticoid benefits thus limiting potential treatment confounders. On the other hand, we recognize that the non-randomized retrospective observational nature of this study limits interpretation of the results and precludes their systematic application in clinical practice. Yet, randomized trials comparing the efficacy of different agents are uncommonly designed especially in the setting of a pandemic, and cohort studies, if appropriately interpreted, can allow for physician thoughtfulness and personalized medicine.

In conclusion, by unveiling a potential window of opportunity to optimize the efficacy of anti IL-1 or IL-6 inhibitors our study provides valuable information not only for interpreting available evidence from past investigations but also for designing future clinical trials.

\section{DATA AVAILABILITY STATEMENT}

The original contributions presented in the study are included in the article/supplementary material. Further inquiries can be directed to the corresponding author.

\section{ETHICS STATEMENT}

The studies involving human participants were reviewed and approved by COVIDBioB Study, Ethical Committee approval no. 34/int/2020, ClinicalTrials.gov NCT04318366. The patients/ participants provided their written informed consent to participate in this study.

\section{AUTHOR CONTRIBUTIONS}

All authors contributed to the article and approved the submitted version. All authors agree to be accountable for all aspects of the 
work in ensuring that questions related to the accuracy or integrity of any part of the work are appropriately investigated and resolved.

\section{COLLABORATIVE CONTRIBUTORS}

BIO-RAF Study Group members: Ada Carla Alba, Elena Baldissera, Costanza Bagnati, Cristina Barberio, Luca Benassi, Nicola Boffini, Enrica P. Bozzolo, Cecilia Bussolari, Stefania Calvisi, Corrado Campochiaro, Giuseppina Maria Casiraghi, Jacopo Castellani, Davide Catarinella, Ludovica Cavallo, Elena Cinel, Giuseppe Dalessandro, Valentina Da Prat, Adriana Cariddi, Antonella Castagna, Giulio Cavalli, Maria Pia Cicalese, Fabio Ciceri, Nicola Compagnone, Lorenzo Dagna, Francesco De Cobelli, Giacomo De Luca, Emanuel Della-Torre, Giuseppe Di Lucca, Gaetano di Terlizzi, Elisabetta Falbo, Nicola Farina, Maria Fazio, Marica Ferrante, Carola Galbiati, Gabriele Gallina, Bruno Nicolò Germinario, Giuseppe Giardina, Giovanni Gobbin,

\section{REFERENCES}

1. Mehta P, McAuley DF, Brown M, Sanchez E, Tattersall RS, Manson JJ, et al. Covid-19: Consider Cytokine Storm Syndromes and Immunosuppression. Lancet (2020) 395:1033-4. doi: 10.1016/S0140-6736(20)30628-0

2. Blanco-Melo D, Nilsson-Payant BE, Liu WC, Uhl S, Hoagland D, Moller R, et al. Imbalanced Host Response to SARS-CoV-2 Drives Development of COVID-19. Cell (2020) 181:1036-45.e9. doi: 10.1016/j.cell.2020.04.026

3. Campochiaro C, Della-Torre E, Cavalli G, De Luca G, Ripa M, Boffini N, et al. Efficacy and Safety of Tocilizumab in Severe COVID-19 Patients: A SingleCentre Retrospective Cohort Study. Eur J Intern Med (2020) 76:43-9. doi: 10.1016/j.ejim.2020.05.021

4. Della-Torre E, Campochiaro C, Cavalli G, De Luca G, Napolitano A, La Marca S, et al. Interleukin-6 Blockade With Sarilumab in Severe COVID-19 Pneumonia With Systemic Hyperinflammation: An Open-Label Cohort Study. Ann Rheum Dis (2020) 79:1277-85. doi: 10.1136/annrheumdis-2020218122

5. Salvarani C, Dolci G, Massari M, Merlo DF, Cavuto S, Savoldi L, et al. Effect of Tocilizumab vs Standard of Care on Clinical Worsening in Patients Hospitalized With COVID-19 Pneumonia: A Randomized Clinical Trial. JAMA Intern Med (2021) 181:24-31. doi: 10.1001/jamainternmed.2020.6615

6. Hermine O, Mariette X, Tharaux P-L, Resche-Rigon M, Porcher R, Ravaud P, et al. Effect of Tocilizumab vs Usual Care in Adults Hospitalized With COVID-19 and Moderate or Severe Pneumonia: A Randomized Clinical Trial. JAMA Intern Med (2020) 181:32-40. doi: 10.1001/jamainternmed. 2020.6820

7. Gupta S, Wang W, Hayek SS, Chan L, Mathews KS, Melamed ML, et al. Association Between Early Treatment With Tocilizumab and Mortality Among Critically Ill Patients With COVID-19. JAMA Intern Med (2020) 181:41-51. doi: 10.1001/jamainternmed.2020.6252

8. Stone JH, Frigault MJ, Serling-Boyd NJ, Fernandes AD, Harvey L, Foulkes AS, et al. Efficacy of Tocilizumab in Patients Hospitalized With Covid-19. N Engl J Med (2020) 383:2333-44. doi: 10.1056/NEJMoa2028836

9. De Luca G, Cavalli G, Campochiaro C, Della-Torre E, Angelillo P, Tomelleri A, et al. Gm-CSF Blockade With Mavrilimumab in Severe COVID-19 Pneumonia and Systemic Hyper-Inflammation: A Single-Centre, Prospective Cohort Study. Lancet Rheumatol (2020) 2:e465-73. doi: 10.1016/S2665-9913(20)30170-3

10. Cavalli G, De Luca G, Campochiaro C, Della-Torre E, Ripa M, Canetti D, et al. Interleukin-1 Blockade With High-Dose Anakinra in Patients With COVID19, Acute Respiratory Distress Syndrome, and Hyperinflammation: A Retrospective Cohort Study. Lancet Rheumatol (2020) 2:e325-31. doi: $10.1016 /$ S2665-9913(20)30127-2
Francesca Guzzo, Giovanni Landoni, Gaetano Lombardi, Marco Lanzillotta, Nicolò Maimeri, Gaia Mancuso, Elena Moizo, Marco Montagna, Giacomo Monti, Luca Moroni, Milena Mucci, Cristina Nakhnoukh, Francesco Giuseppe Nisi, Alessandro Ortalda, Diego Palumbo, Nicola Pasculli, Chiara Pomaranzi, Marco Ripa, Patrizia Rovere-Querini, Annalisa Ruggeri, Silvia Sartorelli, Paolo Scarpellini, Tommaso Scquizzato, Alessandro Tomelleri, Moreno Tresoldi, Alberto Zangrillo.

\section{ACKNOWLEDGMENTS}

The authors wish to thank all the patients who participated in this study and their families, and the healthcare personnel at the investigative site. We are grateful to our own wives, husbands, and kids, patiently waiting at home, physically distant but close to us in supporting our daily work in COVID-19 wards. We dedicate this work to the memory of health care workers who have given their lives in the care of patients with COVID-19.

11. Pontali E, Volpi S, Signori A, Antonucci D, Castellaneta M, Buzzi D, et al. Efficacy of Early Anti-Inflammatory Treatment With High Doses IV Anakinra With or Without Glucocorticoids in Patients With Severe COVID-19 Pneumonia. J Allergy Clin Immunol (2021) 147:1217-25. doi: 10.1016/j.jaci.2021.01.024

12. CORIMUNO-19 Collaborative group. Effect of Anakinra Versus Usual Care in Adults in Hospital With COVID-19 and Mild-to-Moderate Pneumonia (CORIMUNO-ANA-1): A Randomised Controlled Trial. Lancet Respir Med (2021) 9:295-304. doi: 10.1016/S2213-2600(20)30556-7

13. Zangrillo A, Beretta L, Scandroglio AM, Monti G, Fominskiy E, Colombo S, et al. Characteristics, Treatment, Outcomes and Cause of Death of Invasively Ventilated Patients With COVID-19 ARDS in Milan, Italy. Crit Care Resusc (2020) 22:200-11.

14. Ciceri F, Castagna A, Rovere Querini P, De Cobelli F, Ruggeri A, Galli L, et al. Early Predictors of Clinical Outcomes of COVID-19 Outbreak in Milan, Italy. Clin Immunol (2020) 217:108509. doi: 10.1016/j.clim.2020. 108509

15. Della-Torre E, Campochiaro C, Cavalli G, De Luca G, Ciceri F, Zangrillo A, et al. Targeting IL-1, IL-6 or GM-CSF in COVID-19. Response to: 'More Evidences on Which Biologic and Which Pathway is Key in Severe-Critical COVID-19 Pneumonia' by Ferraccioli. Ann Rheum Dis (2020) annrheumdis2020-218612. doi: 10.1136/annrheumdis-2020-218612

16. Della-Torre E, Landoni G, Zangrillo A, Dagna L. Impact of Sarilumab on Mechanical Ventilation in Patients With COVID-19. Response to: 'Correspondence on: 'Interleukin-6 Blockade With Sarilumab in Severe COVID-19 Pneumonia With Systemic Hyperinflammation-an Open-Label Cohort Study' by Della-Torre Et Al' by Cheng and Zhang. Ann Rheum Dis (2020) annrheumdis-2020-218724. doi: 10.1136/annrheumdis-2020-218724

17. Horby P, Lim WS, Emberson JR, Mafham M, Bell JL, Linsell L, et al. Recovery Collaborative Group - Dexamethasone in Hospitalized Patients With Covid19 - Preliminary Report. N Engl J Med (2020) 384:693-704. doi: 10.1056/ NEJMoa2021436

18. Della-Torre E, Della-Torre F, Kusanovic M, Scotti R, Ramirez GA, Dagna L, et al. Treating COVID-19 With Colchicine in Community Healthcare Setting. Clin Immunol (2020) 217:108490. doi: 10.1016/j.clim.2020.108490

19. Della-Torre E, Ramirez GA, Dagna L, Tresoldi M. Colchicine Treatmentin Community Healthcare Setting to Prevent Severe COVID-19. Ann Rheum Dis (2020) annrheumdis-2020-218759. doi: 10.1136/annrheumdis-2020-218759

20. Cavalli G, Larcher A, Tomelleri A, Campochiaro C, Della-Torre E, et al. Effectiveness of Interleukin-1 and Interleukin-6 Inhibition Compared to Standard Management in Patients With COVID-19 and Hyperinflammation: A Cohort Study. Lancet Rheumatol (2021) 3(4):e253-61. doi: 10.1016/S26659913(21)00012-6 
Conflict of Interest: The authors declare that the research was conducted in the absence of any commercial or financial relationships that could be construed as a potential conflict of interest.

Copyright (c) 2021 Della-Torre, Lanzillotta, Campochiaro, Cavalli, De Luca, Tomelleri, Boffini, De Lorenzo, Ruggeri, Rovere-Querini, Castagna, Landoni,
Tresoldi, Ciceri, Zangrillo and Dagna. This is an open-access article distributed under the terms of the Creative Commons Attribution License (CC BY). The use, distribution or reproduction in other forums is permitted, provided the original author(s) and the copyright owner(s) are credited and that the original publication in this journal is cited, in accordance with accepted academic practice. No use, distribution or reproduction is permitted which does not comply with these terms. 